\title{
Embracing the enemy: the diversification of microbial gene repertoires by phage-mediated horizontal gene transfer
}

\author{
Marie Touchon a,b, Jorge A Moura de Sousa ${ }^{a, b}$, Eduardo P.C. Rocha a,b, \\ aMicrobial Evolutionary Genomics, Institut Pasteur, 25-28 rue Dr Roux, Paris, 75015, France \\ ${ }^{\mathrm{b}} \mathrm{CNRS}$, UMR3525, 25-28 rue Dr. Roux, Paris, 75015, France \\ *Author for correspondence: Telephone +33 (0) 1406133 53. Email: erocha@pasteur.fr
}

Short title: Phage-mediated horizontal gene transfer

Keywords: Bacteriophages; horizontal gene transfer; bacterial evolution; mobile genetic elements; transduction.

\begin{abstract}
Bacteriophages and archaeal viruses contribute, through lysogenic conversion or transduction, to the horizontal transfer of genetic material between cellular genomes. Recent works using a variety of approaches have shown that lysogenic conversion is widespread and provides hosts with adaptive traits often associated with biotic interactions. The quantification of the evolutionary impact of transduction has lagged behind and still requires further theoretical and experimental work. Nevertheless, recent studies have suggested that generalized transduction could play a role in the transfer of antibiotic resistance genes and in gene acquisition during intra-specific competition. Phage-mediated horizontal transfer has specific characteristics that complement other mechanisms of transfer. Notably, they could play a key role in the spread of adaptive genes between communities.
\end{abstract}


Horizontal gene transfer (HGT) drives the evolution of the genomes of Prokaryotes. Bacterial phages and archaeal viruses (all called phages henceforth) promote HGT through three major mechanisms (Figure 1). Temperate phages may integrate temporarily the host genome, and the expression of its traits leads to lysogenic conversion [1]. The resulting prophages may excise erroneously and transfer a neighboring piece of the host chromosome in a process called specialized transduction. Finally, host DNA may be packaged by mistake in the capsid during the lytic cycle and lead to generalized transduction of this genetic material to other hosts [2]. The amount of DNA transduced by phages is typically slightly superior to the size of its genome and depends on the internal volume of the capsid [3]. Other mechanisms of HGT implicate virion particles. They are reviewed in other articles of this issue and include transduction by gene transfer agents, the transfer of phages by vesicles, and the subversion of phage virions by genomic islands (see issues in this volume

[TO_ADD_IN_PRODUCTION]).

Both mechanisms of transduction were first reported in the early 1950 's, when phages were essential tools to discover the basic principles of molecular biology. After several decades of less intense study, phages have come back to the spotlight due to increased interest on phage therapies to circumvent antibiotic resistance, to the numerous phage-encoded virulence factors, and because of phages' role in the regulation of microbial populations [4-6]. Transduction and lysogenic conversion are relevant for all these topics. Both processes facilitate the spread of antibiotic resistance and virulence factors. Lysogenic conversion may also protect hosts from other phages. A combination of recent single-cell, genomics, metagenomics (viromes in particular), mathematical modelling, and other techniques has spurred a renewed interest in transduction as a force driving the diversification of microbial populations. This review focus on the most recent works and points to certain limitations in the current knowledge on these topics.

\section{Lysogenic conversion}

Lysogeny often involves the integration of the temperate phage genome in the host chromosome, even if a growing number of prophages are found to replicate in cells as plasmids [7,8]. The expression of prophage genes leads to phenotypic changes in the host that may affect many different traits, including virulence, motility, and inter-bacterial competition (see [5,9] for reviews). The identification of the determinants of the decision between lysis and lysogeny can thus enlighten the role of lysogenic conversion in bacterial evolution.

The decision of lysogeny in temperate phages is taken by a stochastic genetic switch (Figure 1). Lysogeny is result of between $0 \%$ and $50 \%$ of the infections by phage $\lambda$; it is more frequent under high multiplicity of infection (MOI), high host doubling time, and when cells are small $[10,11]$. These 
trends can be explained by the differences between the expected reproduction rate of the element as a prophage and as a lytic phage. Poor host growth conditions lead to small cells, and both lead to low burst sizes, thus reducing the payoff of entering the lytic cycle. High phage concentrations in the environment also reduce the phage reproductive rate because few hosts are available for novel infections. These conditions are expected to favor lysogeny, and this is in agreement with some environmental studies (e.g., see [12]). Two recent phage metagenomics (virome) studies showed that virus-to-microbe ratios decrease with microbial abundance $[13,14]$. Interestingly, the large meta-analysis in [13] also shows that the fraction of temperate phages, among all phages, increases with bacterial cell density. This has spurred the Piggyback-the-winner model, which proposes that lysogeny is favored at high cell densities because phages can attain relatively high reproductive rates from lysogeny when its host is a fast-growing bacterium. It is unclear how this fits the laboratory observations that lysogeny is favored under poor growth conditions, even if it's in accordance with the observations that species able to grow fast under optimal conditions have more prophages (see below, [15]). It could also be argued that high viral densities result in low phage reproductive rates, and thus favor lysogeny. But how could phages take an informed decision on lysogeny based on the environmental concentration of phages? High multiplicity of infection is a proxy of phage density in the environment and shifts the decision towards lysogeny in phage $\lambda$ [11]. Alternatively, some phages encode mechanisms resembling quorum-sensing that allow the infecting phage to sense the environmental concentration of phages and use this information to decide between lysis and lysogeny [16]. Other molecular mechanisms may provide phages with information valuable for the decision of lysogeny and contribute to explain its frequency under high viral concentrations.

Changes in community composition complicate the meta-analysis of virome data because the frequency of temperate phages depends on traits beyond the joint dynamics of lysogeny and prophage induction. Notably, it depends on the bacterial species present in the community. Around half of the sequenced genomes harbor a prophage [17]. Whereas some species have extremely large numbers of prophages, e.g., close to 20 in certain Escherichia coli strains, others have none, e.g., no prophage could be found in the genomes of 21 Chlamydia trachomatis strains [15]. The contribution of prophages to the diversity of gene repertoires can be huge: they accounted for $41 \%$ of the accessory genome of a set of $47 \mathrm{E}$. coli strains [18]. Prophages are more frequent in bacteria with larger genomes (and may be a cause or a consequence of the latter) and in those that are pathogens (in line with evidence showing that many virulence factors are encoded in prophages) [15]. But, somewhat surprisingly, the species minimum doubling time was the strongest statistical determinant of lysogeny identified in a recent analysis [15], suggesting a strong link between copiotrophic lifestyles and lysogeny. This is consistent with environmental data showing that 
lysogeny depends on the variability of prokaryote physiology [19]. Phages infecting bacteria with strong variations in growth rates, i.e., those with high maximal growth rates or strong population bottlenecks, may face more variable benefits from lysis and thus engage more often in lysogeny.

During lysogeny there is an alignment of interests between prophages and hosts because their reproductions are tightly linked. This alignment can be disrupted in several ways. First, the induction of the prophage under situations of endangered host viability, leads to phage reproduction at the cost of cell death (Figure 1). Hence, the adaptive traits carried by lysogenic conversion come at a significant risk for the host [20]. Second, mutations may inactivate the prophage and reduce its ability to kill the host. In this context, it of interest to know the viability of the prophages, which has been studied exhaustively in two studies. Only two of the 18 E. coli 0157:H7 Sakai prophages, and only one of the seven Enterococcus faecalis V583 prophages, were found to produce viable infectious particles. Hence, most prophages were defective at some level. In theory, the existence of recombination between phages and prophages [21-23], could permit the reanimation of defective prophages by purging their inactivating mutations. However, since prophages tend to prevent infection by closely related phages, the probability of reanimation shortly after the first inactivation events might be small (and even lower afterwards, when many mutations have accumulated). In spite of the near irreversibility of prophage inactivation, many prophages in the two abovementioned studies were shown to be inducible, produce virions, and/or were able to lyse the host cell $[24,25]$. There are even reports of groups of prophages coordinating their induction [21]. Hence, defective prophages may remain dangerous genetic elements even when their replication becomes almost exclusively dependent on host reproduction.

Given that inactivated prophages can still kill the host, it might be thought that natural selection would quickly remove them from the population. Yet, defective prophages may encode adaptive traits [26], and remain in genomes for long periods of time, during which inactivating mutations are counter-selected [27]. Domestication of degraded prophages may thus result in long-standing lysogenic conversion of the host. Furthermore, the constant influx of novel prophages facilitates the processes of co-optation of their molecular machineries, leading to functional innovation (see $[28,29]$ for reviews).

\section{Specialized transduction}

Specialized transduction results from an event of inaccurate excision of the prophage from the chromosome, e.g., by "illegitimate" recombination, that leads to the packaging of a section of the prophage and the contiguous chromosomal DNA (Figure 1). Illegitimate recombination depends on the local density of repeats [30], with the consequence that specialized transduction rates may be 
very susceptible to the chromosomal context of the prophage. Specialized transduction occurs at very low frequency in $\lambda\left(10^{-6}\right.$ relative to normal excision [31]), and has been described in few other phages (e.g., [32,33]). Even though quantitative data is lacking, it is usually thought that most transducing phages are defective in certain functions. Specialized transduction can thus be a oneshot event, much like generalized transduction (see below), and integration of the genetic information in the novel cellular host genome may depend on the homologous recombination machinery. Nevertheless, the internal volume of the capsid allows packaging a DNA molecule that is longer than the phage genome, up to $108 \%$ in $\lambda$ [34], and specialized transduction may sometimes lead to the transfer of complete, or almost complete, phages (at the cost of transducing very little bacterial DNA). The relevance of specialized transduction to bacterial evolution is difficult to quantify experimentally and by comparative genomics, explaining the paucity of recent literature on the subject (outside the specific domain of genetic engineering [35]).

\section{Generalized transduction}

Generalized transduction results from errors in discriminating phage from chromosomal DNA during packaging. This mechanism can transfer any chromosomal sequence, including rDNA [36], and its integration in the host chromosome may require homologous recombination. Generalized transduction has usually been identified in phages packaging their genome using the pac system [37]. Yet, many phages seem to be able to transduce DNA, e.g., a study has identified that $99 \%$ of Salmonella prophages were capable of generalized transduction [38]. Furthermore, some mobile elements that parasite phage capsids seem to have evolved the ability to be packaged by cos phages (using this other major mechanism of DNA packaging) [39].

Until recently, it was thought that generalized transduction occurred at low frequency. However, recent single-cell analyses observed transduction rates close to $1 \%$ per plaque forming units when natural communities were used as recipients [40]. The differences from previous works may result from genetic and environmental effects that affect the rate of generalized transduction. For example, transduction rates are low under high phage density, because the host of the transducing phage is likely to be infected (and killed) by viable phages. Generalized transduction is a concern during phage therapy, since it may transfer virulence or resistance determinants between cells [41]. Temperate phages engaging in generalized transduction can be used to both kill competitors and obtain their genes. This works in two steps. Lysis of a small fraction of the population of lysogens generates phages that can decimate competing populations of sensitive cells [42]. This should be advantageous during colonization, even if only lasts as long as the initially sensitive population does not become lysogenic (and thus phage resistant) $[43,44]$. The reproduction of the phage in the 
sensitive population leads to generalized transduction of their genes to the original bacterial population. Hence, lysogens can use their prophages to kill other bacteria and then use the resulting transducing virions to acquire novel genes. This mechanism of autotransduction depends on lysogens that are protected against super-infection and accept transducing virions. In the laboratory, it was shown to greatly increase the rate of transfer of antibiotic resistance genes to Staphylococcus aureus [45].

\section{Determinants of transfer}

Many variables affect phage-mediated HGT, and further theoretical work is needed to understand how they could interact in transmission networks (Box 1). The phage host range affects the ability of these processes to spread genes in communities. The analysis of the networks of gene homology between bacteria and phages (so-called transduction networks) suggests that most transfer takes place between closely related taxa [46], in agreement with the traditional view that phages have narrow host ranges. Yet, this view is being challenged by the discovery of elements able to cross the species or even the genus barrier [6,47-49]. The consequences of these findings are amplified by the observation that transduction host-range is often broader than the phage replication host-range $[50,51]$, and that host range is best described as a continuum of decreasing ability to infect a given host [52]. An extreme example of this is given by the proposal that eukaryotic-like proteins in a phage of Wolbachia result from inter-kingdom transduction events [53]. Such phages, even if rare, might have key roles in the transmission of genes across phyla.

Phage-mediated HGT depends on the efficiency of bacterial defense tools, which have been very intensely studied in the last few years and cannot be reviewed in this short article (see [54]). Phages encode their own tools to circumvent bacterial defenses [55]. They also encode tools to protect their prophages (and the host) from infections by other viruses [56]. A recent study has searched systematically for these protective systems in cluster $\mathrm{N}$ mycobacteriophages and identified five distinct systems that interfere with closely related or unrelated phages [57]. All these systems are expected to affect the rates of transfer between individual cells. For example, bacteria with compatible restriction-modification systems have been found to engage in much higher rates of HGT and homologous recombination than the others [58].

\section{Antibiotic resistance}

The role of transduction in the spread of antibiotic resistance genes (ARG) is a relatively recent topic of research because these genes are much often identified in conjugative elements than in phages. However, work in this topic is being spurred by studied identifying many ARG in gut viromes 
following antibiotic perturbation $[59,60]$. The analysis of viromes is technically challenging because bacterial contamination can be mistaken by transducing phages [61]. Yet, a number of reports also identified ARG in bona fide phages or prophages (e.g., [62,63]), or in transducing elements [64]. These observations suggest that phages could play an important role in the transmission of ARG from external to host-associated environments because phage-mediated HGT can take place between distant bacterial cells [65]. The actual effect of antibiotic treatments in transduction is complex and requires further theoretical treatment because sub-lethal doses of antibiotics can stimulate the induction of the lytic cyle in lysogens in vivo [66], and increase the efficiency of phage infection [67]. Furthermore, the presence of phages can offset the costs of antibiotic resistance by increasing selection for high mutation rates in hosts [68]. The scientific and clinical relevance of this topic deserve further attention in the future.

\section{Conclusions}

Much remains to be known regarding the role of phages in HGT (Box 2). Even if metagenomics data suggests that generalized transduction may contribute significantly to HGT in prokaryotes, quantitative data on this process is still lacking. Our ignorance is even more dramatic regarding the significance of the contribution of specialized transduction, whose role in prokaryotic evolution remains to be demonstrated. At this stage, the relevance of lysogeny in HGT is well-established, but novel studies and approaches are needed to understand the relevance of transduction.

Mathematical models may pave the way to integrate the existing knowledge on the population dynamics of phages and bacteria, and guide future work on phage-mediated HGT (see Box 1).

The specific characteristics of the three mechanisms of phage-mediated HGT may affect microbial evolutionary dynamics. First, these mechanisms differ in terms of the number and diversity of chromosomal genes they can transfer. Second, they are driven by different types of phages: specialized transduction is restricted to temperate integrative phages, lysogenic conversion to temperate phages, and generalized transduction is usually observed in pac-phages. Third, host defence systems affect these mechanisms differently. In particular, CRISPR-Cas systems target phage DNA and are thus expected to be more permissive to generalized transduction. Fourth, although all mechanisms are affected by the phage host range, transduction events requiring homologous recombination to integrate the chromosome may be more affected by host range than lysogenic conversion. Finally, prophages may be induced and kill their hosts. On larger evolutionary scales these distinctions can be blurred by recombination between virulent and temperate phages [69], but at the population scale one expects that phage-mediated HGT will depend strongly on the characteristics of the phages and bacteria present in the community. 
Relative to other mechanisms of HGT, like transformation and conjugation, phage-mediated HGT has some unique traits. On the downside, transduction occurs at low rates and typically involves few chromosomal genes, which may restrict its ability to transfer complex traits (requiring the cotransfer of many genes). Specialized transduction and lysogeny may also result in host death. On the upside, phage-mediated transfer does not require direct cell-to-cell contact (like conjugation), and can transfer DNA across environments (unlike transformation). Phages can survive in the environment for long periods of time, allowing time-delayed transfer of genetic information. They could thus play instrumental roles in spreading traits between communities. Further theoretical and empirical work is urgently needed to understand the range of conditions favouring phage-mediated transfer and their consequences to the evolution of cellular genomes. 


\section{Box 1| Modelling transduction of adaptive traits}

Models are simplified representations of reality that can be used to predict the outcomes of biological processes and, when they fail, pinpoint deficiencies in our current knowledge. Theoretical approaches can establish the expected temporal dynamics of processes, and the study of bacteriaphage interactions has benefited greatly from the development of mathematical models. These have been used, for instance, to study growth and predation dynamics [70], the evolution of CRISPRs [71], the consequences of phage therapy [72], or the use of cell suicide as a defence from phages [73]. However, there were very few attempts at modelling phage-mediated transmission of adaptive traits between bacteria. A possible reason for the dearth of such approaches might be the complexity of integrating bacterial adaptation, predator-prey behaviours, and within-host viral dynamics.

The relative importance of generalized and specialized transduction for the dissemination of an adaptive trait is unclear. Theoretical models could be used to explore plausible dynamics, providing insights and experimentally testable hypotheses. This has been shown for other mechanisms of horizontal gene transfer [74] and can therefore also be used to disclose the impact of transduction in the adaptation of bacterial populations. A recent study used a system of deterministic differential equations to model the spread of an adaptive trait by transduction, and estimated that both generalized and specialized transduction might have a minor role in the spread of antibiotic resistance genes relative to conjugation [75].

However, the interplay between ecology and evolution, involving community and environmental structure, can significantly impact the dynamics of transduction. Individual-based models (IBMs) are an emerging modelling approach that provides a suitable framework to study these and other dynamics in microbial systems [76]. IBMs provide a link between individual heterogeneity (e.g., bacteria infected by different phages, or at different stages of infection) and population-level dynamics (e.g., the frequency of horizontally transferred adaptive traits or the community composition), whilst accounting for stochasticity. Thus, they could become a useful tool in understanding the role of phages in the evolution of prokaryotes' gene repertoires. 
Box 2| Some outstanding questions:

- What is the frequency with which lysogenic conversion is adaptive?

- What are the ranges and rates of generalized transduction?

- What is the evolutionary relevance of specialized transduction?

- What are the relative roles of physiology, phage and host density in the decision of lysogeny?

- Are there specific evolutionary roles for the different modes of phage-mediated HGT?

- What is the evolutionary relevance of the mechanism of auto-transduction? 


\section{Figure captions}

Figure 1. Major phage-mediated HGT mechanisms

Phages contribute to bacterial evolution by generating genetic diversity through horizontal gene transfer (HGT). Infection by temperate phages can lead to either the lytic cycle (represented in green) or the lysogenic cycle (represented in blue). Infection by virulent phages leads to the lytic cycle. Transduction occurs when newly forming phages acquire host genes and transfer them to other bacterial cells. (A) Generalized transduction can transfer random fragments of host or plasmid DNA into other phage sensitive cells. It occurs when phage packaging accidentally incorporates host instead of phage DNA (red). It can be done by both temperate and virulent phages. (B) Specialized transduction is due to faulty excision of the prophage from the host chromosome leading to a phage genome with both phage (red) and host genes (black). This type of transducing phage only transfers host genes neighbouring the integration site of prophage, and is restricted to temperate phages. (C) Lysogenic conversion occurs when a prophage (integrated or episomal) induces a change in the phenotype of the infected cell (that is not part of the phage lifecycle). For example, prophages may encode proteins such as toxins (red circles), that are either secreted, or released on bacterial lysis, and contribute to bacterial pathogenicity. (D) Some molecular machineries (orange), such as killer particles, R-type pyocins (tailocins), gene transfer agents (GTAs) and type VI secretion systems (T6SSs), are structurally, and one assumes evolutionarily, related to phage components. GTAs are non-replicative phage-like elements integrated in the host chromosome that package and transduce random host DNA fragments for constitutive transduction. 


\section{Acknowledgements}

Work in our laboratory is funded by credits from the CNRS and the Pasteur Institute. We thank Mireille Ansaldi for comments on a previous version of this manuscript, and Louis-Marie Bobay and Aude Bernheim for discussions.

\section{References}

1. Canchaya C, Proux C, Fournoux G, Bruttin A, Brussow H: Prophage genomics. Microbiol Mol Biol Rev 2003, 67:238-276.

2. Thierauf A, Perez G, Maloy AS: Generalized transduction. Methods Mol Biol 2009, 501:267-286.

3. De Paepe M, Taddei F: Viruses' Life History: Towards a Mechanistic Basis of a Trade-Off between Survival and Reproduction among Phages. PLoS Biol 2006, 4:e193.

4. Pirnay JP, De Vos D, Verbeken G, Merabishvili M, Chanishvili N, Vaneechoutte M, Zizi M, Laire G, Lavigne R, Huys I, et al.: The phage therapy paradigm: pret-a-porter or sur-mesure? Pharm Res 2011, 28:934-937.

5. Bondy-Denomy J, Davidson AR: When a virus is not a parasite: the beneficial effects of prophages on bacterial fitness. J Microbiol 2014, 52:235-242.

6. Koskella B: Phage-mediated selection on microbiota of a long-lived host. Curr Biol 2013, 23:12561260.

7. Brolund A, Franzen O, Melefors O, Tegmark-Wisell K, Sandegren L: Plasmidome-analysis of ESBLproducing escherichia coli using conventional typing and high-throughput sequencing. PLoS One 2013, 8:e65793.

8. Xue H, Cordero OX, Camas FM, Trimble W, Meyer F, Guglielmini J, Rocha EP, Polz MF: EcoEvolutionary Dynamics of Episomes among Ecologically Cohesive Bacterial Populations. MBio 2015, 6:e00552-00515.

9. Obeng N, Pratama AA, Elsas JD: The Significance of Mutualistic Phages for Bacterial Ecology and Evolution. Trends Microbiol 2016, 24:440-449.

**10. St-Pierre F, Endy D: Determination of cell fate selection during phage lambda infection. Proc Natl Acad Sci U S A 2008, 105:20705-20710.

A pioneering work on the determinants of lysogeny using single-cell approaches. See also [11].

11. Zeng L, Skinner SO, Zong C, Sippy J, Feiss M, Golding I: Decision making at a subcellular level determines the outcome of bacteriophage infection. Cell 2010, 141:682-691.

12. Pradeep Ram AS, Sime-Ngando T: Resources drive trade-off between viral lifestyles in the plankton: evidence from freshwater microbial microcosms. Environ Microbiol 2010, 12:467479.

** 13. Knowles B, Silveira CB, Bailey BA, Barott K, Cantu VA, Cobian-Guemes AG, Coutinho FH, Dinsdale EA, Felts B, Furby KA, et al.: Lytic to temperate switching of viral communities. Nature 2016, 531:466-470.

An impressive metagenomics and meta-analysis of metagenomics data showing the association between phage and bacteria concentration in marine environments. The results suggest that lysis is 
suppressed where established models predict it should be favored. Proposes the Piggyback-theWinner model.

* 14. Wigington $\mathrm{CH}$, Sonderegger D, Brussaard CPD, Buchan A, Finke JF, Fuhrman JA, Lennon JT, Middelboe M, Suttle CA, Stock C, et al.: Re-examination of the relationship between marine virus and microbial cell abundances. Nature Microbiology 2016, 1:15024.

Shows that the very cited ratio of one bacteria for 10 phages in the environment is incorrect. The phage-to-bacteria ratio decreases with the increase in bacterial density and shows large variations depending on the environment.

15. Touchon M, Bernheim A, Rocha EP: Genetic and life-history traits associated with the distribution of prophages in bacteria. ISME J 2016, 10:2744-2754.

Quantifies the frequency of lysogens and the number of prophages in bacterial genomes (see also [17]). It determines the variables significantly associated with lysogeny or prophage abundance, including maximal growth rates, pathogenicity, length of CRISPR arrays, and genome size and those that are not significantly associated, notably cell volume, optimal growth temperature, and presence of CRISPR-Cas systems.

** 16. Erez Z, Steinberger-Levy I, Shamir M, Doron S, Stokar-Avihail A, Peleg Y, Melamed S, Leavitt A, Savidor A, Albeck $\mathrm{S}$, et al.: Communication between viruses guides lysis-lysogeny decisions. Nature 2017, 541:488-493.

The spectacular identification of a phage-encoded quorum-sensing mechanism that evaluates the environmental density of similar phages to decide between lysis and lysogeny. Contributes to explain why phages favor lysogeny when viral densities are high.

** 17. Roux S, Hallam SJ, Woyke T, Sullivan MB: Viral dark matter and virus-host interactions resolved from publicly available microbial genomes. Elife 2015, 4:e08490.

Shows that around half of the bacteria are lysogens (see also [13]), and identifies a large number of novel viral families, confirming that cross-taxon recombination between phages is relatively rare. It introduces the software VirSorter (along with [75]).

18. Bobay L-M, Rocha EPC, Touchon M: The Adaptation of Temperate Bacteriophages to Their Host Genomes. Mol Biol Evol 2013, 30:737-751.

19. Maurice CF, Bouvier C, de Wit R, Bouvier T: Linking the lytic and lysogenic bacteriophage cycles to environmental conditions, host physiology and their variability in coastal lagoons. Environ Microbiol 2013, 15:2463-2475.

20. Paul JH: Prophages in marine bacteria: dangerous molecular time bombs or the key to survival in the seas? ISME J 2008, 2:579-589.

21. Lemire S, Figueroa-Bossi N, Bossi L: Bacteriophage crosstalk: coordination of prophage induction by trans-acting antirepressors. PLoS Genetics 2011, 7:e1002149.

* 22. De Paepe M, Hutinet G, Son O, Amarir-Bouhram J, Schbath S, Petit MA: Temperate phages acquire DNA from defective prophages by relaxed homologous recombination: the role of Rad52-like recombinases. PLoS Genet 2014, 10:e1004181. 
Phages may acquire genetic information from prophages by recombination. The effect seems to be particularly important for phages encoding Rad52-like recombinases, which are more permissive to sequence divergence.

23. Menouni R, Hutinet G, Petit MA, Ansaldi M: Bacterial genome remodeling through bacteriophage recombination. FEMS Microbiol Lett 2015, 362:1-10.

24. Asadulghani M, Ogura Y, Ooka T, Itoh T, Sawaguchi A, Iguchi A, Nakayama K, Hayashi T: The defective prophage pool of Escherichia coli 0157: prophage-prophage interactions potentiate horizontal transfer of virulence determinants. PLoS Pathogens 2009, 5:e1000408.

25. Matos RC, Lapaque N, Rigottier-Gois L, Debarbieux L, Meylheuc T, Gonzalez-Zorn B, Repoila F, Lopes Mde F, Serror P: Enterococcus faecalis prophage dynamics and contributions to pathogenic traits. PLoS Genet 2013, 9:e1003539.

26. Wang X, Kim Y, Ma Q, Hong SH, Pokusaeva K, Sturino JM, Wood TK: Cryptic prophages help bacteria cope with adverse environments. Nat Commun 2010, 1:147.

* 27. Bobay LM, Touchon M, Rocha EPC: Pervasive domestication of defective prophages by bacteria. Proc Natl Acad Sci U S A 2014, 111:12127-12132.

Shows that many prophages accumulate significantly fewer non-synonymous substitutions that expected, suggesting widespread domestication of these elements by the host.

28. Veesler D, Cambillau C: A common evolutionary origin for tailed-bacteriophage functional modules and bacterial machineries. Microbiol Mol Biol Rev 2011, 75:423-433.

29. Touchon M, Bobay LM, Rocha EP: The chromosomal accommodation and domestication of mobile genetic elements. Curr Opin Microbiol 2014, 22:22-29.

30. Rocha EPC: An appraisal of the potential for illegitimate recombination in bacterial genomes and its consequences: from duplications to genome reduction. Genome Res 2003, 13:11231132.

31. Gottesman ME, Yarmolinsky MB: Integration-negative mutants of bacteriophage lambda. J Mol Biol 1968, 31:487-505.

32. Fernandes RM, de Lencastre $\mathrm{H}$, Archer LJ: Specialized transduction in Bacillus subtilis by the phages IG1, IG3, and IG4. Arch Virol 1989, 105:137-140.

33. Campos J, Martinez E, Marrero K, Silva Y, Rodriguez BL, Suzarte E, Ledon T, Fando R: Novel type of specialized transduction for CTX phi or its satellite phage RS1 mediated by filamentous phage VGJ phi in Vibrio cholerae. J Bacteriol 2003, 185:7231-7240.

34. Feiss M, Fisher RA, Crayton MA, Egner C: Packaging of the bacteriophage lambda chromosome: effect of chromosome length. Virology 1977, 77:281-293.

35. Jain P, Hsu T, Arai M, Biermann K, Thaler DS, Nguyen A, Gonzalez PA, Tufariello JM, Kriakov J, Chen $\mathrm{B}$, et al.: Specialized transduction designed for precise high-throughput unmarked deletions in Mycobacterium tuberculosis. MBio 2014, 5:e01245-01214.

36. Beumer A, Robinson JB: A broad-host-range, generalized transducing phage (SN-T) acquires $16 \mathrm{~S}$ rRNA genes from different genera of bacteria. Appl Environ Microbiol 2005, 71:8301-8304.

37. Oliveira L, Tavares P, Alonso JC: Headful DNA packaging: bacteriophage SPP1 as a model system. Virus Res 2013, 173:247-259. 
38. Schicklmaier P, Moser E, Wieland T, Rabsch W, Schmieger H: A comparative study on the frequency of prophages among natural isolates of Salmonella and Escherichia coli with emphasis on generalized transducers. Antonie Van Leeuwenhoek 1998, 73:49-54.

39. Chen J, Carpena N, Quiles-Puchalt N, Ram G, Novick RP, Penades JR: Intra- and inter-generic transfer of pathogenicity island-encoded virulence genes by cos phages. ISME J 2015, 9:1260-1263.

** 40. Kenzaka T, Tani K, Nasu M: High-frequency phage-mediated gene transfer in freshwater environments determined at single-cell level. ISME J 2010, 4:648-659.

A single-cell study showing unexpectedly high levels of generalized transduction in freshwater environments.

41. Matilla MA, Fang X, Salmond GP: Viunalikeviruses are environmentally common agents of horizontal gene transfer in pathogens and biocontrol bacteria. ISME J 2014, 8:2143-2147.

42. Bossi L, Fuentes JA, Mora G, Figueroa-Bossi N: Prophage contribution to bacterial population dynamics. J Bacteriol 2003, 185:6467-6471.

* 43. Gama JA, Reis AM, Domingues I, Mendes-Soares H, Matos AM, Dionisio F: Temperate bacterial viruses as double-edged swords in bacterial warfare. PLoS One 2013, 8:e59043.

A study exploring the conditions upon which prophages might be used by bacteria to eliminate competitors.

* 44. Davies EV, James CE, Kukavica-Ibrulj I, Levesque RC, Brockhurst MA, Winstanley C: Temperate phages enhance pathogen fitness in chronic lung infection. ISME J 2016, 10:2553-2555.

Prophages may increase the competitiveness of the host in chronic lung infections by reducing competing populations of sensitive bacteria.

** 45. Haaber J, Leisner JJ, Cohn MT, Catalan-Moreno A, Nielsen JB, Westh H, Penades JR, Ingmer H: Bacterial viruses enable their host to acquire antibiotic resistance genes from neighbouring cells. Nat Commun 2016, 7:13333.

An intriguing study showing that bacteria may use prophages to outcompete competitors and recover their genetic information by autotransformation.

46. Popa O, Landan G, Dagan T: Phylogenomic networks reveal limited phylogenetic range of lateral gene transfer by transduction. ISME J 2016, 11:543-554.

47. Jensen EC, Schrader HS, Rieland B, Thompson TL, Lee KW, Nickerson KW, Kokjohn TA: Prevalence of broad-host-range lytic bacteriophages of Sphaerotilus natans, Escherichia coli, and Pseudomonas aeruginosa. Appl Environ Microbiol 1998, 64:575-580.

48. Sullivan MB, Waterbury JB, Chisholm SW: Cyanophages infecting the oceanic cyanobacterium Prochlorococcus. Nature 2003, 424:1047-1051.

49. Hyman P, Abedon ST: Bacteriophage Host Range and Bacterial Resistance. Adv Appl Microbiol 2010, 70:217-248.

50. Chiura HX: Generalized gene transfer by virus-like particles from marine bacteria. Aquatic Microbial Ecology 1997, 13:75-83.

51. Chen J, Novick RP: Phage-mediated intergeneric transfer of toxin genes. Science 2009, 323:139141. 
52. Koskella B, Meaden S: Understanding bacteriophage specificity in natural microbial communities. Viruses 2013, 5:806-823.

* 53. Bordenstein SR, Bordenstein SR: Eukaryotic association module in phage WO genomes from Wolbachia. Nat Commun 2016, 7:13155.

Wolbachia phages encode eukaryotic-like proteins. They might result from inter-kingdom HGT.

54. Labrie SJ, Samson JE, Moineau S: Bacteriophage resistance mechanisms. Nat Rev Microbiol 2010, 8:317-327.

55. Samson JE, Magadan AH, Sabri M, Moineau S: Revenge of the phages: defeating bacterial defences. Nat Rev Microbiol 2013, 11:675-687.

56. Bondy-Denomy J, Pawluk A, Maxwell KL, Davidson AR: Bacteriophage genes that inactivate the CRISPR/Cas bacterial immune system. Nature 2013, 493:429-432.

** 57. Dedrick RM, Jacobs-Sera D, Bustamante CA, Garlena RA, Mavrich TN, Pope WH, Reyes JC, Russell DA, Adair T, Alvey R, et al.: Prophage-mediated defence against viral attack and viral counter-defence. Nat Microbiol 2017, 2:16251.

A very thorough study of several novel mechanisms allowing prophages to protect themselves (and their hosts) from other phages.

** 58. Oliveira PH, Touchon M, Rocha EP: Regulation of genetic flux between bacteria by restriction-modification systems. Proc Natl Acad Sci U S A 2016, 113:5658-5663.

Shows that diversification of defense systems (here, type II restriction-modification systems) affects the intra-species flux of genetic material. Compatible cells engage in much higher rates of HGT and homologous recombination than incompatible ones.

** 59. Modi SR, Lee HH, Spina CS, Collins JJ: Antibiotic treatment expands the resistance reservoir and ecological network of the phage metagenome. Nature 2013, 499:219-222.

This study provides evidence for the role of phages in the evolution and spread of antibiotic resistance between bacteria of mice microbiota.

60. Brown-Jaque M, Calero-Caceres W, Muniesa M: Transfer of antibiotic-resistance genes via phage-related mobile elements. Plasmid 2015, 79:1-7.

61. Enault F, Briet A, Bouteille L, Roux S, Sullivan MB, Petit MA: Phages rarely encode antibiotic resistance genes: a cautionary tale for virome analyses. ISME J 2017, 11:237-247.

62. Iannelli F, Santagati M, Santoro F, Oggioni MR, Stefani S, Pozzi G: Nucleotide sequence of conjugative prophage Phi1207.3 (formerly Tn1207.3) carrying the mef(A)/msr(D) genes for e ffl ux resistance to macrolides in Streptococcus pyogenes. Front Microbiol 2014, 5:687.

63. Billard-Pomares T, Fouteau S, Jacquet ME, Roche D, Barbe V, Castellanos M, Bouet JY, Cruveiller $\mathrm{S}$, Medigue C, Blanco J, et al.: Characterization of a P1-like bacteriophage encoding an SHV2 extended-spectrum beta-lactamase from an Escherichia coli strain. Antimicrob Agents Chemother 2014, 58:AAC.03183-03114.

64. Goh S, Hussain H, Chang BJ, Emmett W, Riley TV, Mullany P: Phage varphic2 mediates transduction of Tn6215, encoding erythromycin resistance, between Clostridium difficile strains. MBio 2013, 4:e00840-00813. 
65. Muniesa M, Colomer-Lluch M, Jofre J: Could bacteriophages transfer antibiotic resistance genes from environmental bacteria to human-body associated bacterial populations? Mob Genet Elements 2013, 3:e25847.

66. Allen HK, Looft T, Bayles DO, Humphrey S, Levine UY, Alt D, Stanton TB: Antibiotics in feed induce prophages in swine fecal microbiomes. MBio 2011, 2:00260-00211.

67. Comeau AM, Tetart F, Trojet SN, Prere MF, Krisch HM: Phage-Antibiotic Synergy (PAS): betalactam and quinolone antibiotics stimulate virulent phage growth. PLoS One 2007, 2:e799.

68. Tazzyman SJ, Hall AR: Lytic phages obscure the cost of antibiotic resistance in Escherichia coli. ISME J 2015, 9:809-820.

69. Labrie SJ, Moineau S: Abortive infection mechanisms and prophage sequences significantly influence the genetic makeup of emerging lytic lactococcal phages. J Bacteriol 2007, 189:1482-1487.

70. Levin BR, Stewart FM, Chao L: Resource-limited growth, competition, and predation: a model and experimental studies with bacteria and bacteriophage. The American Naturalist 1977, 111:3-24.

71. Koonin EV, Wolf YI: Evolution of the CRISPR-Cas adaptive immunity systems in prokaryotes: models and observations on virus-host coevolution. Mol Biosyst 2015, 11:20-27.

72. Levin BR, Bull JJ: Population and evolutionary dynamics of phage therapy. Nat Rev Microbiol 2004, 2:166-173.

* 73. Berngruber TW, Lion S, Gandon S: Evolution of suicide as a defence strategy against pathogens in a spatially structured environment. Ecol Lett 2013, 16:446-453.

A study on the conditions where bacterial suicide upon being infected by a phage is adaptive.

74. Niehus R, Mitri S, Fletcher AG, Foster KR: Migration and horizontal gene transfer divide microbial genomes into multiple niches. Nat Commun 2015, 6:8924.

** 75. Volkova VV, Lu Z, Besser T, Grohn YT: Modeling the infection dynamics of bacteriophages in enteric Escherichia coli: estimating the contribution of transduction to antimicrobial gene spread. Appl Environ Microbiol 2014, 80:4350-4362.

A deterministic mathematical model of transduction of an antibiotic resistance gene. The model is used to estimate the contribution of transduction to horizontal gene transfer, which is inferred to be around 1000 times lower than that of conjugation.

* 76. Hellweger FL, Clegg RJ, Clark JR, Plugge CM, Kreft JU: Advancing microbial sciences by individual-based modelling. Nat Rev Microbiol 2016, 14:461-471.

A perspective on the uses of individual-based modelling in microbiology, including applications to phage biology.

77. Roux S, Enault F, Hurwitz BL, Sullivan MB: VirSorter: mining viral signal from microbial genomic data. PeerJ 2015, 3:e985. 


\section{Highlights}

- Phages drive horizontal gene transfer between prokaryotes.

- Lysogenic conversion provides novel adaptive traits, at the cost of eventual lysis.

- Prophages account for a sizeable fraction of bacterial gene repertoires.

- The environmental rates of specialized and generalized transduction, and their evolutionary relevance, are poorly known.

- Theoretical work is necessary to understand the potential implication of transduction in the evolutionary dynamics of prokaryotes. 

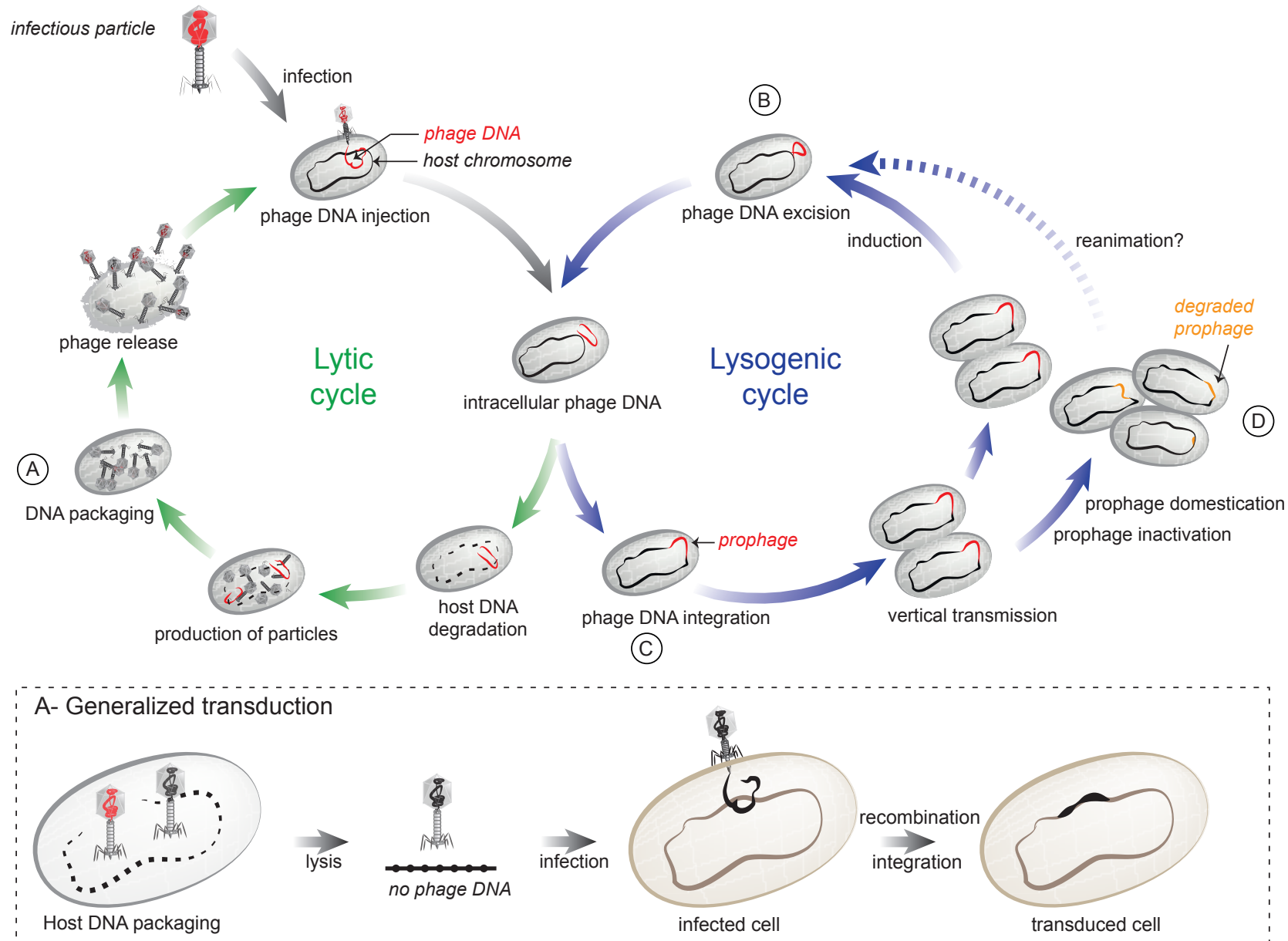

B- Specialized transduction
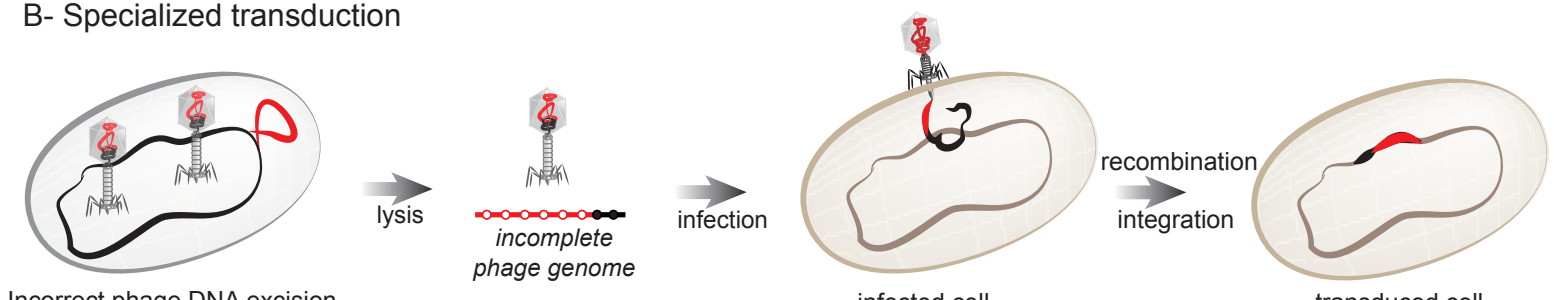

Incorrect phage DNA excision

infected cell

transduced cell

C- Lysogenic conversion
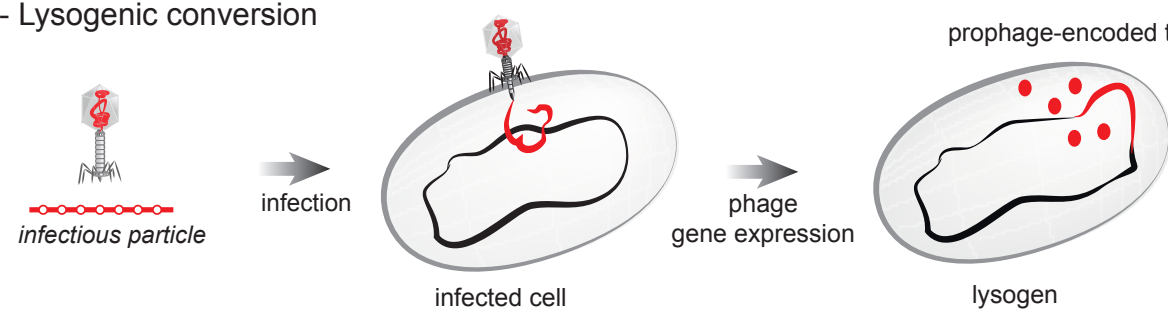

lysogen

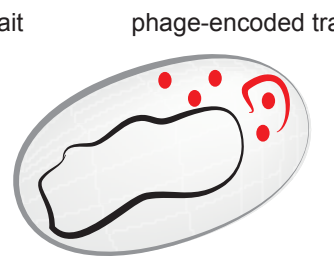

lysogen

D- Phage-related elements

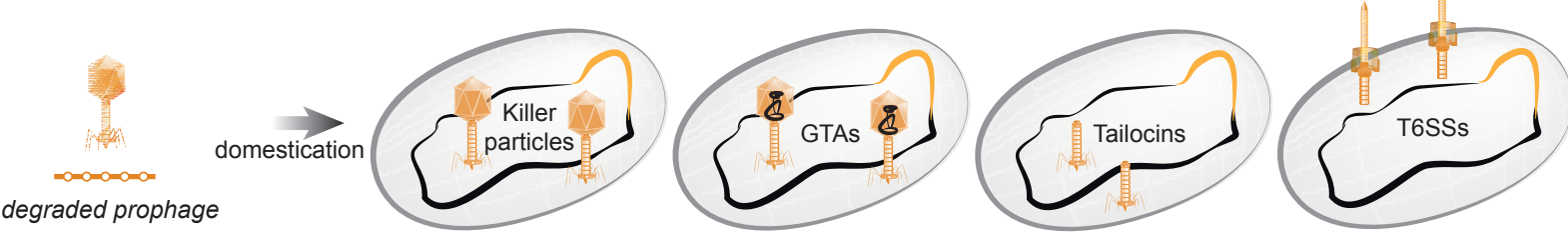
degraded prophage 


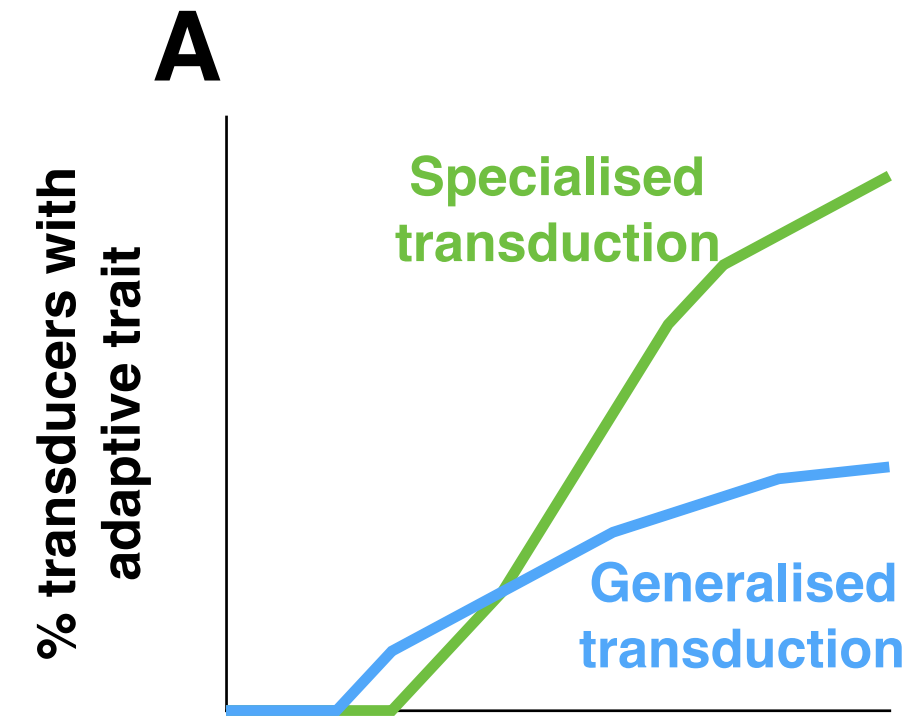

Time
B

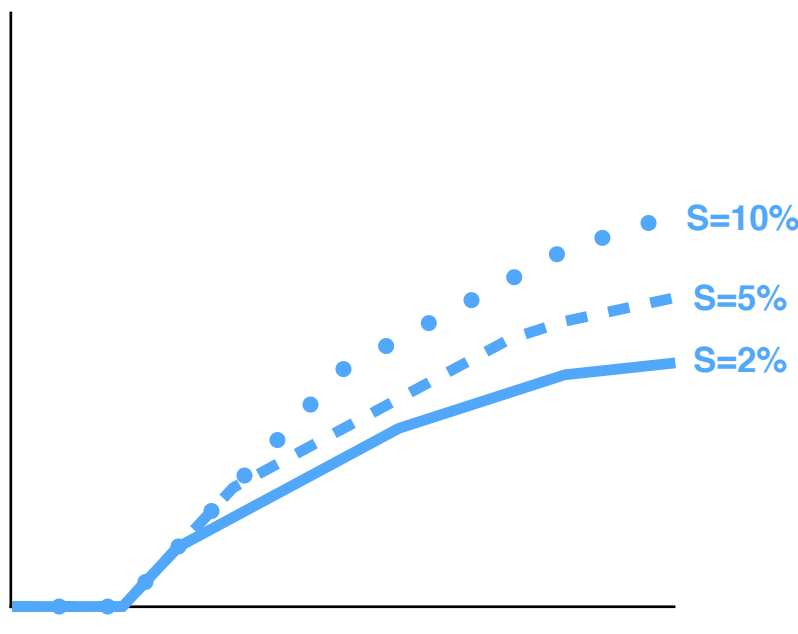

Time
C

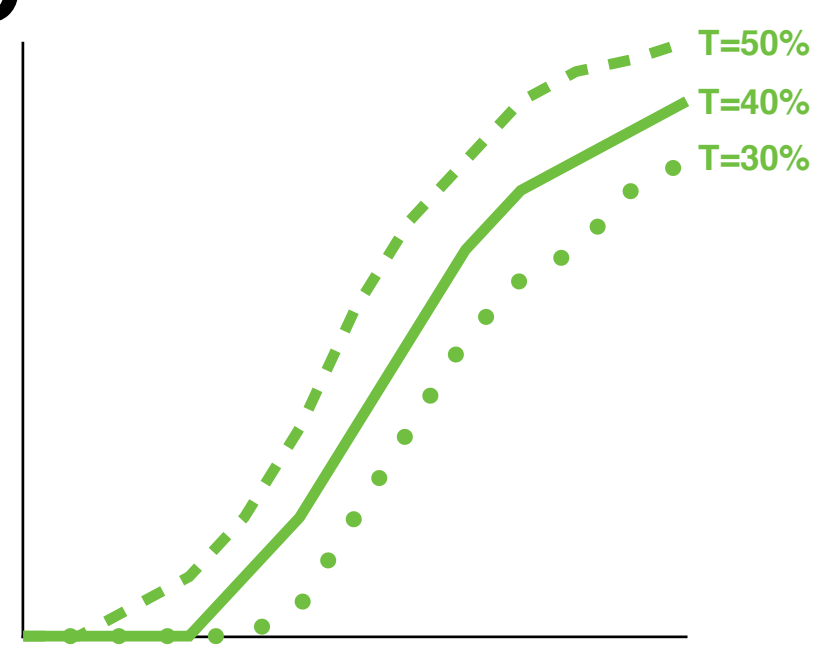

Time

Hypothetical dynamics of specialised and generalised transduction in the spread of an adaptive trait. (A) Generalised transduction (blue line) could be more likely to incorporate the adaptive trait that provides an advantage in this scenario, since it has potential access to any gene in the bacterial genome. However, its further spread is limited to vertical inheritance, since generalised transduction generates defective phage. Conversely, specialised transduction (green line) of a specific adaptive trait is less likely to occur (since encapsulation involves solely genes nearby the phage integration site), but its dissemination can be much faster, since the phage retain their viral characteristics. Hence, dissemination of the adaptive trait is enhanced by infection dynamics. (B) The vertical inheritance of defective phages where generalised transduction has occurred is mainly determined by the selective advantage $(S)$ provided by the transduced trait. We hypothesise that, in a structured environment, the spread of an adaptive trait by vertical transmission is slower than in a mixed (liquid) environment. (C) If the trait is acquired by viable phage through specialised transduction, then its rate of dissemination of in a bacterial population could be mostly defined by the phage transmissivity $(T)$. This compound variable represents the ability of phage to reach, infect and integrate new bacterial hosts, thus providing an horizontal path to the dissemination of the adaptive trait. 